\title{
1 Introduction to the book
}

\author{
JEAN P. PALUTIKOF ${ }^{1}$, SARAH L. BOULTER ${ }^{1}$, \\ JON BARNETT2 AND DAVID RISSIK ${ }^{1}$ \\ ${ }^{1}$ National Climate Change Adaptation Research Facility, Griffith University, Australia \\ ${ }^{2}$ School of Geography, University of Melbourne, Australia
}

\subsection{Why this book exists}

\subsubsection{NCCARF and its research programs}

In 2008 , in response to a clearly felt need to build national resilience and capacity to adapt to climate change, the Australian Government funded the National Climate Change Adaptation Research Facility (NCCARF) for a period of 5 years and for $\$ 50$ million. The mission of NCCARF was to generate and deliver to Australian decision-makers the knowledge needed to effectively adapt to climate change. Of the $\$ 50$ million, a sum of $\$ 30$ million was used to fund research projects into adaptation, making this one of the largest such research programs anywhere in the world at that time. It resulted in some 120 projects, exploring every aspect of adaptation relevant to Australia.

\subsubsection{How was the research program constructed?}

The research program was constructed around groups of sectors, namely:

- marine biodiversity and resources;

- terrestrial and freshwater biodiversity;

- primary industries;

- settlements and infrastructure;

- human health;
- emergency management

- adaptation and Indigenous communities; and

- social, economic and institutional dimensions of adaptation.

As a first step, and for each topic, a team of researchers and practitioners in adaptation was brought together to write a research plan that identified knowledge gaps and research priorities. Each research plan went through an intensive period of consultation and review, to ensure it properly reflected the views of the adaptation community. The identified research priorities then became the basis for an open call for proposals.

In addition to these thematic topics, a crossdiscipline synthesising and integrative topic was constructed. This consisted of short projects (rarely longer than 12 months) based mainly around literature reviews and stakeholder interviews, identified as being of particular interest to practitioners, sometimes in response to real-world events. Thus NCCARF funded a number of floodrelated adaptation research projects in response to questions asked by stakeholders following the widespread devastating floods that affected large parts of Australia in 2010 and again in 2011. A number of chapters in this volume are based around these synthesis and integrative research projects.

A notable feature of the research program was the involvement of end-users. As emphasised by

Applied Studies in Climate Adaptation, First Edition. Edited by Jean P. Palutikof, Sarah L. Boulter, Jon Barnett and David Rissik. (C) 2015 John Wiley \& Sons, Ltd. Published 2015 by John Wiley \& Sons, Ltd. 
the title of this book, adaptation is essentially an applied field of study (Chapter 2 by Palutikof et al.). NCCARF's adaptation research aimed to be useful to the end-user community and to be delivered in accessible formats. To address this requirement, project investigators were required to write and fulfil an end-user communication plan and/or to set up a project steering committee that included strong participation from end-users.

\subsubsection{Why this book?}

All the final reports from the NCCARF research programs in its first phase are available on the website www.nccarf.edu.au. These are often weighty volumes, containing the detail of the relevant literature, the research methodology and analyses of extensive datasets. NCCARF has already devoted considerable effort to producing user-friendly summaries of the research findings, and continues to do so. However, these summaries are primarily directed towards Australian adaptation practitioners; they assume considerable contextual knowledge, and focus on the concerns and problems raised by adaptation in Australia.

As outlined in Chapter 2 of this volume, Australia has much experience in adapting to climate variability and change. It has one of the most (naturally) variable climates in the developed world, and already the impacts of anthropogenic climate change are being experienced. Australian decision-makers face a multiplicity of issues as they seek timely and cost-effective responses to climate change which balance the needs and concerns of different stakeholder groups, while ensuring that planning, regulatory and legislative requirements are met. These issues, and the emerging solutions to address them, are not particular to Australia. To name just a few in the area of primary industry, this book deals with how farmers are adapting to changing conditions (Chapter 14 by Hertzler et al. and Chapter 15 by Kingwell et al.), the likely outcomes of the interaction of irrigation water tariffs and markets with climate change (Chapter 17 by Cooper et al. and Chapter 18 by Wheeler et al.), and how value chains in the fishing and aqua- culture industries can evolve profitably under climate change (Chapter 16 by Hobday et al.).

The editors of this book (and the authors of this chapter) see much value in sharing the results from NCCARF's research programs with the wider global community of adaptation researchers and end-users. In preparing their chapters, all authors were asked to consider the international context for their work and the international implications of their results.

\subsection{Structure and content of the book}

After this introductory chapter, the book continues with a chapter written by the editors which highlights some of their emerging thoughts and conclusions around adaptation, and how these have been influenced during the editorial process. These are presented as four loosely linked arguments. First, we argue that the Australian experience with adaptation policy and research is of global relevance. Second, we can no longer say that adaptation to climate change is not happening, even if we might disagree about its effectiveness. Third, there is limited utility, and probably only confusion, in the increasing classification of types of adaptation, for example as being 'autonomous' or 'evolutionary' in nature. Fourth, we argue that adaptation is not a 'science' as sometimes claimed, but rather a complex interdisciplinary 'field of inquiry' whose ultimate rationale must include delivering policy-relevant information to decision-makers.

The chapters that follow are divided into nine sections. To increase the international relevance of the book, we approached nine (non-Australian) experts in adaptation and asked them to contribute short 'think pieces' to open each of these nine sections. We would like to express our gratitude to these nine experts and thank them for entering into the spirit of the exercise. We gave them little in the way of guidance, simply emphasising that these should be their musings on the subject of their section, based on their experiences over the years.

The first section covers frameworks for enabling adaptation and looks at the context 
within which adaptation takes place and the instruments and circumstances that can act to facilitate or impede the adaptation process. Gary Yohe from the United States contributes the think-piece, providing some personal reflections. Chapter 4 (McAneney) looks at the role of insurance, concluding that the short time horizons of the industry impede its capacity to support adaptation to climate change. Macintosh and colleagues (Chapter 5) look at planning instruments for adaptation, and how these can be designed to facilitate adaptation. This is followed by a chapter on public perceptions of climate change by Reser et al. Chapter 7 (Verdon-Kidd et al.) then explores the science-policy interface and the challenges faced by those who seek to cross that interface.

This introductory framing section is followed by eight thematic sections. These are arranged in a semi-logical order, building from the natural environment through to settlements, disaster management and business. Inevitably, there is overlap between sections. For example, a number of chapters deal with heat stress and adaptation; these can be found in Section 5 on building resilience among vulnerable groups (Chapter 27 by Hansen et al.), in Section 6 on the Indigenous experience of climate change (Chapter 32 by Horne and Martel) and in Section 7 on settlements and housing (Chapter 39 by Saman et al. and Chapter 40 by Barnett et al.).

The thematic section on ecosystem management (Section 2) is opened by a think-piece by Jonathan Overpeck with the opening question: at what level does climate change pose an 'unacceptable' risk to biodiversity? There follow four chapters on adaptation management options for birds (Chapter 9 by Franklin et al.), seabirds and marine mammals (Chapter 10 by Hobday et al.), Ramsar wetlands (Chapter 11 by Bino et al.) and finally invertebrates (Chapter 12 by Moir).

Section 3 on farming has a think-piece from John Morton making the point that, although Australia is a highly developed economy, it is also (and unusually so for a developed country) largely tropical and subtropical; several features of its agriculture therefore have resonance for some poorer countries of the tropics. The five main chapters in this section explore climate change impacts and adaptation interactions for broadacre (dryland) farming (Chapter 14 by Hertzler et al.; Chapter 15 by Kingwell et al.), irrigation water governance (Chapter 17 by Cooper et al.; Chapter 18 by Wheeler et al.) and value chains in the fisheries and aquaculture industries (Chapter 16 by Hobday et al.).

Section 4 on coasts opens with a think-piece by Susi Moser. This is a personal reflection on the stresses, both human and physical, which affect the coastal zone and a call to arms to all those researchers and decision-makers with responsibilities in the coastal zone to ensure that the coastal zone remains a place of beauty and sustenance. Four chapters follow. Woodroffe and colleagues (Chapter 20) show how climate change can be integrated into risk assessment approaches and combined with engineering models to create more comprehensive coastal risk assessments. Hadwen and Capon (Chapter 21) also present a risk-based approach to adaptation, focusing on habitats and ecosystems and emphasising the need to consider non-climatic pressures when exploring adaptation options. McEvoy and Mullett (Chapter 22) have a specific interest in climate change risks to seaports and their operation. Fletcher and colleagues (Chapter 23) explore the economics, equity and institutional arrangements for adaptation. Chapter 24 by Waters and Barnett concludes this section, exploring the views of stakeholders on where the responsibilities lie for adaptation in the coastal zone.

Section 5 covers the building of resilience among vulnerable groups. The think piece is an essay by Hallie Eakin on the 'turn' that has taken place from a focus on vulnerability to climate change, to a focus on capacity. In Chapter 26, Barnett and Palutikof explore the limits to adaptation using five example locations in Australia where the impacts of future climate change may exceed the capacity to adapt. There follow two chapters on extreme events, looking at how the impacts on vulnerable groups are experienced and can be managed. Chapters 27 (Hansen et al.) and 28 (Correa-Velez et al.) look, respectively, at the effects of heat waves on culturally and linguistically diverse groups and at 
the experience of the 2011 Queensland floods by refugee groups. Sevoyan and Hugo (Chapter 29) explore the relationships between social exclusion and vulnerability to climate change, and how the barriers of social exclusion can be overcome to ensure that effective adaptation can take place. Finally, in Chapter 30 Hamilton and Mallon look at the vulnerability of community organisations to extreme events, and the implications on their capacity to continue to provide community services under climate change.

Section 6 is on the Indigenous experience of climate change. The think-piece by Meg Parsons places the challenge of climate change to Australia's Indigenous communities firmly within a global context. It proposes two requirements for Indigenous communities to prosper under climate change: the first is to move beyond current conceptualisation of Indigenous as 'traditional' and consider the diversity of Indigenous communities; the second relates to future planning and the need to consider how adaptation relates to social justice and Indigenous rights. There follow four diverse chapters considering adaptation and Indigenous communities from four very different perspectives. Chapter 32 (Horne and Martel) looks at Indigenous housing in the desert climate of Alice Springs, and at what modifications may be required to ensure a level of comfort under an even hotter climate. Haynes and colleagues explore the Indigenous experience of Cyclone Tracy, which razed Darwin to the ground in December 1974, in Chapter 33. The authors seek to understand whether there were attributes of the Indigenous experience which might point to particular capacities and vulnerabilities. In Chapter 34 Tran and colleagues look at the role of native title in the governance of adaptation, using two small Indigenous settlements as case studies. Nursey-Bray and colleagues (Chapter 35) look at adaptation in one Indigenous group (the Arabana people) and, in particular, how their attachment to and engagement with their land influences their capacity to adapt.

Section 7 on settlements and housing opens with a think-piece by Bill Solecki on global urban development and climate change. There follow five chapters in this section; Chapters 37 and 38 are on settlements and Chapters 39-41 are on housing. Beer and colleagues (Chapter 37) develop an index of vulnerability for country towns, which suggests that it is the most remote communities that are the most vulnerable. Mortazavi-Naeini and colleagues test the robust multi-objective optimisation approach as a tool to assist urban water supply managers to adapt to uncertain future climate change in Chapter 38. Saman and colleagues (Chapter 39) look at how design can improve the comfort levels of houses during heat waves. Barnett and colleagues (Chapter 40) also explore thermal performance in heat waves, but with a particular emphasis on low-income housing. Finally, Instone and colleagues (Chapter 41) look at strategies for facilitating adaptation in the rental housing market, which again in Australia is primarily a low-income market.

Section 8 is on the topic of adaptation and disaster management. The think-piece is provided by Ian Burton, reflecting on the shortcoming of 'practical adaptation' and the need to ensure that responses to disasters - responses he terms 'palliative adaptation' - do not leave us more vulnerable to long-term climate change. Three chapters follow. In Chapter 43, Boon seeks to understand the factors that promote community resilience in the face of disasters by studying four towns and four different events: bushfire, flood, drought and cyclone. She finds that strong individual resilience and social connectedness promote strong community resilience. Bird and colleagues describe a similar study in Chapter 44 but in this case focused on flood disasters, looking at the respective roles of individuals, communities and government in responding to disasters and the factors that promote and inhibit resilience. Howes (Chapter 45) completes this section by investigating the potential for governments to conserve scarce resources by integrating disaster risk management and climate change adaptation.

The final section is on the subject of business. It opens with a think-piece by Frans Berkhout exploring organisational adaptation from the perspectives of utility-maximising, behavioural and institutional approaches. Shearer and colleagues (Chapter 47) look at major barriers and drivers 
of adaptive capacity for the urban property development industry, noting that moves towards greater sustainability are primarily driven by client demand which, in Australia at least, is generally lacking. Kuruppu and colleagues investigate adaptation among small-to-medium enterprises (SMEs) in Chapter 48. These authors conclude that SMEs, where they adopt adaptation strategies, address climate extremes rather than climate change, and that it is their past experience of extremes that is their primary motivator for adaptation. Finally, in Chapter 49 West proposes a framework to enable businesses to better consider the strengths and weaknesses of various approaches for assessing adaptation options, and includes a case study of wind damage to coastal assets.
Acknowledgements

We would like to acknowledge financial support from the Australian Government through the Department of Climate Change and Energy Efficiency, without which it would not have been possible to produce this book. Most chapters are based on research funded through this initiative. We would also like to thank all those who have worked in the Research Management Team of NCCARF over the years: Richard McKellar (Research Manager), Florence Crick, Ida Fellegara, Daniela Guitart, Ann Penny, Frank Stadler, David George and Daniel Stock. Their diligence in ensuring quality in the NCCARF research projects is carried through into this book. 